\section{Microperimetry in age: related macular degeneration}

E Midena ${ }^{1,2}$ and E Pilotto ${ }^{1}$

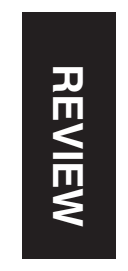

\begin{abstract}
Age-related macular degeneration (AMD) is one of the major causes of visual loss and legal blindness in people over 55 . Visual function tests are the cornerstone of visual function investigation and any therapeutic approach to AMD implies, as primary endpoint, the maintenance or improvement of visual function. The progression of visual impairment and the quantification of final residual visual function are currently determined by means of visual acuity quantification. The quantification of high-contrast visual acuity though has many drawbacks and cannot be considered a complete functional examination. Microperimetry is a non-invasive method used to analyse fixation and central visual field defects in a topographic related manner. The introduction of mesopic and more recently scotopic microperimetry, in research and clinical practice of macular disorders, now allows us to better investigate macular function as it strictly relates to macular morphology. We therefore can monitor the functional natural history and quantify the beneficial or detrimental effects of different therapies. The application of microperimetry in clinical studies has provided interesting diagnostic and prognostic information on functional macular changes in AMD patients. The present review brings new updates on the correlation between macular changes, mainly described with optical coherence tomography, and microperimetry changes in patients with AMD.

Eye (2017) 31, 985-994; doi:10.1038/eye.2017.34; published online 3 March 2017
\end{abstract}

\section{Introduction}

Even if far and near distance visual acuity (VA) do not seem to be affected in the early phases of age-related macular degeneration (AMD), many patients report alterations in their visual perception, mainly in low luminance light conditions. ${ }^{1,2}$ The progression of AMD toward the more advanced phases invariably involves the foveal area which in turn shows a marked and irreversible decrease of the retinal function and the development of dense and irreversible scotomas. This progression may also be functionally described by the instability and loss of retinal fixation. Moreover, in the natural history of early AMD, rod photoreceptors degenerate earlier and more rapidly than cones. ${ }^{3,4}$ The greatest loss in rod function occurs in the para-foveal region of the macula, which extends from 3.5 to $10^{\circ}$ from the center of the fovea, where rod density is at its maximum. ${ }^{4}$ Despite the evidence that rods fail first, clinicians and regulatory agencies monitor the progression of macular degeneration with visual tests that measure cone, rather than rod function. This is typically performed in a bright light background, but not in dim light conditions, where patients struggle to perform ordinary visual tasks. Therefore, a more comprehensive approach to quantify macular function in AMD should be encouraged. Microperimetry is a noninvasive method used to analyse fixation and central retinal sensitivity.

The introduction of mesopic and more recently scotopic microperimetry, in research and clinical practice of macular disorders allows us to better investigate macular function as it strictly relates to macular morphology. The application of microperimetry in clinical studies has provided interesting diagnostic and prognostic information on macular functional changes in AMD patients.

The present review summarizes the most recent investigations on the correlation between morphological macular changes and retinal sensitivity data and the role of microperimetry in routine clinical practice in patients suffering from AMD.

\section{Microperimetry: the technologic evolution}

Microperimetry (also known as fundusperimetry) is the diagnostic technique which allows us to exactly correlate, in real time, the
${ }^{1}$ Department of Ophthalmology, University of Padova, Padova, Italy

${ }^{2} \mathrm{~GB}$ Bietti Foundation, IRCCS, Rome, Italy

Correspondence: E Midena, Department of Ophthalmology, University of Padova, via Giustiniani 2, Padova 35128, Italy Tel: +39 498212110 ; Fax: +39498755168 . E-mail: edoardo.midena@ unipd.it

Received: 7 July 2016 Accepted in revised form: 18 January 2017 Published online: 3 March 2017 
sensitivity threshold of any individual point of the retina with its clinical (biomicroscopic and OCT) appearance. It also documents the location and stability of fixation. Microperimetry was first introduced when scanning laser ophthalmoscope (SLO) allowed us, for the first time, to easily and reliably document the above mentioned morpho-functional correlation. ${ }^{5,6}$ The original SLO (Rodenstock, Germany), which is no more commercially available, was the first instrument combining static perimetric testing and simultaneous observation of the fundus. SLO microperimetry allowed a real-time examination, by means of an infrared light source of the retina and allowed a manual projection of visual stimuli of different shapes, sizes and intensities over selected retinal areas. ${ }^{5}$ A sensitivity map, expressed in $\mathrm{dB}$ or pseudocolors, was then available at the end of the examination. SLO microperimetry however, did not allow for fully automatic examinations and follow-up. Moreover, it never really underwent major hardware and software improvements, which then in turn led to its limited diffusion in clinical practice.

Twenty years later, in the early 2000 s, a fully automatic microperimeter was introduced into the clinical practice (Micro Perimeter 1, MP1, Nidek, Japan). This instrument allowed for fast and reliable functional fundus-related examination of fixation and scotoma characteristics in patients affected by retinal and choroidal diseases, with topographic accuracy and test-retest reliability, even when visual acuity was poor and fixation was unstable and eccentric. ${ }^{7-10}$ The development of this microperimeter has dramatically expanded the application of microperimetry in clinical practice, providing relevant information on functional changes in several macular and extramacular disorders. ${ }^{11,12}$ MP1 software contains an automatic tracking system of eye movements. This system evaluate in each acquired frame the $X$ and $Y$ shifts of the eye compared to a baseline reference frame and continuously corrects stimulus location according to the current fundus position. This is performed in real time during the acquisition. Stimulus intensity may be varied in $1 \mathrm{~dB}$ steps ( $0.1 \log$ steps) between $0 \mathrm{~dB}\left(400 \mathrm{asb}, 127 \mathrm{~cd} / \mathrm{m}^{2}\right)$, the instrument's maximum stimulus luminance, and $20 \mathrm{~dB}\left(4 \mathrm{asb}, 1.27 \mathrm{~cd} / \mathrm{m}^{2}\right)$, the minimum stimulus luminance. The stimulus size may be varied according to standard Goldmann stimulus sizes: from I to V (from 6.5 to $104 \mathrm{~min} / \mathrm{arc}$ ).

Fixation is continuously registered during a microperimetry examination assessing retinal dynamic fixation, but it may also be recorded as an isolated fixation task (static fixation). At the end of any microperimetry test, the acquired fixation points are exactly superimposed over a color fundus photography, taken by the embedded CCD camera. The software of the microperimeter automatically analyses fixation stability according to two different methods: the clinical classification proposed by Fujii et al, and the bivariate contour ellipse area analysis. ${ }^{13-15}$

Retinal sensitivity of every single retinal tested point is reported in $\mathrm{dB}$ or pseudocolors. An interpolated pseudocolors map of macular sensitivity may also be created. These data may be reported onto an infrared image, or onto a fundus color photography. The interpolated pseudocolors map may be useful to graphically compare microperimetry map with OCT map. Moreover, the MP1 software allows a graphic representation of other perimetric indices, particularly the automatic evaluation of an individual map versus agerelated thresholds.

Using the MP1 microperimeter software, any retinal image of the same eye, acquired with any different technique (fluorescein and indocyanine green angiography, fundus autofluorescence picture, and so on), may imported and overlapped onto the sensitivity map. This allows for an integrated imaging approach in different macular diseases.

More recently, new microperimeters have become available. One is an LCD microperimeter, with the same background and stimulation characteristics as the MP1 microperimeter combined with a SLO/OCT (SLO/OCT, Opko, USA). With this system it is possible to overlap the mean retinal sensitivity map (interpolated map) with an infrared image or a SLO/OCT map. Unfortunately, it lacks the color fundus photograph, thus the overlap of retinal sensitivity and anatomical details or an OCT thickness map is mainly an integration of mean values.

Another instrument is the SLO microperimeter (MAIA, Centervue, Italy), with improved imaging compared to the old SLO microperimeter, wider sensitivity range (0$34 \mathrm{~dB}$ compared to $0-20 \mathrm{~dB}$ od the MP1) and a very efficient software. Normative age-related data are available, and its reproducibility has been tested. ${ }^{16,17}$ However, it has no possibility of overlapping onto a color fundus photograph (except when taken with a different instrument).

The new generation of the MP-1, the MP-3 (Nidek, Gamagori, Japan), provides a wider range of stimulus intensities (0-34 dB).

MP1 has been recently modified to perform rod threshold measurements. The MP1 scotopic microperimeter (MP-1S) has new hardware and software. It is a modified version of the standard MP-1 and allows the examiner to perform localized rod sensitivity measurements of dark-adapted patients in a darkroom environment. Therefore, MP-1S operates in two visual function conditions: one optimized for light adapted eyes in an examination room that has mesopic light conditions ('mesopic use'), and the other optimized for use in dark adapted eyes in a darkroom environment ('scotopic use'). 


\section{Microperimetry in early age-related macular degeneration}

Soft intermediate drusen $(>63 \mu \mathrm{m})$ and areas of hyperpigmentation and/or hypopigmentation of the retinal pigment epithelium (RPE) are the earliest manifestations of AMD. Aging RPE cells show an agerelated accumulation of lipofuscin granula within the lysosomal compartment, as a product of the permanent phagocytosis of lipid-rich distal photoreceptors' outer segments. ${ }^{18}$ Lipofuscin, responsible for the in vivo fundus autofluorescence phenomenon (FAF), is considered a biomarker of cellular aging and a cumulative index of oxidative damage. ${ }^{19}$ It is believed that lipofuscin accumulation precedes photoreceptor degeneration, which is functionally represented by abnormal retinal sensitivity in central retinal area, and slower rates of dark adaptation. ${ }^{20-24}$ Histopathologic studies of eyes with AMD have identified cell death, structural, compositional, and transcriptional modifications and morphologic changes in photoreceptor cells that directly overlie drusen. ${ }^{25-27}$ Deflection and shortening of rod and cone inner and outer segments with consequent retinal thickness reduction have been confirmed..$^{25}$ Using Spectral Domain OCT a decrease in the thickness of photoreceptor layer has been confirmed in vivo. ${ }^{28}$ Decrease in retinal sensitivity correlates with the location of drusen or RPE changes. ${ }^{29,30}$ When correlating retinal sensitivity above drusen with retinal structural changes, visualized with spectral domain OCT, the photoreceptor inner/outer-segment (IS/OS) junction, now better known as ellipsoid zone, has a predictive value for retinal sensitivity changes. ${ }^{31}$ Retinal sensitivity reduction develops much earlier than visual acuity changes, and it happens in a short time. ${ }^{17,30,32,33}$

Reticular pseudodrusen is an additional specific clinical hallmark of AMD. ${ }^{34}$ This macular lesion, also known as reticular or cuticular drusen or subretinal drusenoid deposit (SDD), has been recognized as an independent risk factor for AMD progression. Moreover, SDD is responsible for the development of a particular, recently described, phenotype of late AMD, called outer retinal atrophy, which is mainly characterized by the atrophy of the photoreceptor layer. ${ }^{35}$ From a functional point of view, both distance and near visual acuity are not significantly different between eyes with SDD and those without it. Otherwise, low-luminance visual acuity measurement is more compromised in eyes with SDD compared with those without it. ${ }^{36}$ Similarly to soft drusen, the distribution and number of SDD correlate with the reduced mean retinal sensitivity in the macular area. ${ }^{37}$ As SDD distribution is more widespread than drusen, the retinal sensitivity is also more widely reduced than in eyes with drusen alone. ${ }^{38}$
Recently, both scotopic and mesopic microperimetry have been investigated in patients with AMD and welldemarcated areas of SDD. ${ }^{39}$ In the same eye, scotopic and mesopic retinal sensitivity over SDD-involved retinal areas and over areas with no visible pathologic changes have been compared. Interestingly, over areas with SDD a reduction in retinal sensitivity for both mesopic and scotopic threshold values, have been detected. Moreover, scotopic scotoma was denser than mesopic scotoma. ${ }^{39}$ This result is in accordance with histologic findings of a preferential vulnerability of the rod system and relative preservation of cone photoreceptors in the early stages of AMD. ${ }^{4,27}$

\section{Geographic atrophy}

Geographic atrophy (GA) represents the atrophic latestage manifestation of AMD. ${ }^{40} \mathrm{GA}$, which is commonly bilateral, is characterized by the development of areas of RPE and neural retina atrophy, which slowly progresses over time. ${ }^{41-44}$ Areas of GA are characterized by a dense scotoma, whose extention corresponds to the atrophic area. ${ }^{45}$ Therefore, the progression of GA is associated with progressive loss of visual function. ${ }^{41}$

Atrophic areas initially occur in the parafoveal retina, and patients are unaware of their functional condition. Over time, several atrophic areas may coalescence, and new atrophic areas may occur. In more advanced stages, the atrophic areas form an atrophic ring around the fovea, which can remain unaffected by the atrophic enlargement for a long time, a phenomenon known as foveal sparing. ${ }^{45,46}$ As long as the fovea remains unaffected, retinal fixation remains central and stable. This means that even a small residual area of retinal sensitivity is useful for fixation in patients with progressive atrophic lesions. ${ }^{47}$

Sunness et al studied fixation patterns in eyes with GA and central scotoma in detail. ${ }^{48}$ Using SLO microperimetry, these authors investigated preferred retinal locus (PRL) in 41 eyes of 35 patients with GA who had lost foveal function. ${ }^{48}$ Sixty-three percent of GA eyes fixating outside the atrophy placed the scotoma to the right of fixation in visual field space providing the best functional result. This phenomenon is partly readingdriven, to prevent that the left-to right reader has the scotoma projected over the beginning of the text. The second adaptation choice, which is also visual cortex controlled, was to have the scotoma above fixation (in $22 \%$ of the cases). ${ }^{49}$

Progressive enlargement of GA is usually assessed by color fundus images or, more recently, by fundus autofluorescence (FAF). ${ }^{41,42,46}$ Short-wavelength FAF (SW-FAF, excitation $488 \mathrm{~nm}$, emission $>500 \mathrm{~nm}$ ) is an imaging method that allows topographic mapping of 
lipofuscin distribution in the retinal pigment epithelium (RPE) in vivo. ${ }^{19,50,51}$ Recently, near-infrared FAF (NIRFAF, excitation $787 \mathrm{~nm}$, emission $>800 \mathrm{~nm}$ ) has been introduced to visualize the distribution of melanin, a fluorophore of the RPE cells and choroid. ${ }^{52}$ Because of the absence of RPE, atrophic areas in GA show a severely reduced FAF signal, appearing hypo-autofluorescent (hypo-FAF) both at SW-FAF and NIR-FAF imaging. Therefore, such areas may be easily quantified using these imaging techniques. ${ }^{46,53}$

Dense scotoma at microperimetry corresponds to hypofluorescent areas at FAF. Moreover, an abnormal autofluorescence pattern in the junctional zone of GA (the area between atrophy and normal retina, on FAF) has been demonstrated using confocal SLO. Areas of increased FAF in the junctional zone seem to precede the enlargement of pre-existing atrophy and the development of new atrophic ares over time. ${ }^{50}$

Fine matrix visual field documented a severe reduction in scotopic sensitivity and moderate reduction in photopic sensitivity over junctional hyperfluorescent regions. ${ }^{54}$ Using SLO microperimetry, reduced sensitivity has been documented in areas of increased FAF bordering GA. ${ }^{55}$ This finding suggests that pathologic lipofuscin accumulation in the RPE surrounding areas of GA has reduced retinal sensitivity as a functional correlate. Moreover, retinal sensitivity reduction has also been detected in areas of decreased or increased NIR-FAF. ${ }^{53,56}$ It may be speculated that the impairment of photoreceptors determines increased NIR-FAF, due to increased phagocytosis and melanogenesis, followed by reduced NIR-FAF due to a decline of melanosomes. ${ }^{57}$

Recently, it has been shown that GA can be both identified (with high-reproducibility) and quantified (with high-repeatability) by means of en face OCT. ${ }^{58,59}$ As a complement to conventional B-scan OCT, en face OCT is a new approach for fundus imaging. It provides a coronal full macular view at different depth levels, and provides additional anatomic insights about macular changes. Recent studies characterized microperimetry of GA as identified from en face OCT imaging. In fact Hariri et al, evaluating retinal sensitivity in the junctional zone and beyond, observed a precipitous drop in sensitivity at the GA margin, than a gradual transition. ${ }^{60}$ This finding was unexpected considering Portella et al study. ${ }^{59}$ Indeed, these authors detected an area of ellipsoid zone disruption on en face OCT surrounding GA areas, that seemed to foreshadow areas of future GA growth. Considering this finding, it would be expected that this disrupted areas may be associated with a mild progressive reduction in mesopic retinal sensitivity. However, en face OCT images vary according to the different position of the coronal scan, in the retina or in the choroid, and GA area is larger when visualized at the outer retina $(\mathrm{OR})$ level than at the choroidal $(\mathrm{CH})$ level. $^{58}$ Pilotto et al recently observed that GA areas, visualized on en face OCT at the OR level and at the $\mathrm{CH}$ level, differ not only for extension, but also for retinal sensitivity. ${ }^{61}$ In eyes with better residual sensitivity, and smaller mean GA area, the mean rate of relative or dense scotoma was different between GA visualized with the two en face images. In $\mathrm{CH}$ en face OCT image, $66 \%$ of the stimulated points were defined as dense scotoma, while on OR en face OCT image the rate of dense scotoma was $52.30 \% .{ }^{61}$ These findings suggest that the GA area visualized at choroidal level better represents the worst functioning retinal zones (dense scotomas), whereas the larger GA area, visualized at outer retina level, corresponds to the wider functionally compromised retina. Therefore, in the early stages of GA, areas identified by en face OCT at the OR level, correctly mirror the functional degenerative involvement of the photoreceptors, suggesting an earlier degenerative involvement of the outer retina and RPE than the underlying, longer surviving choriocapillaris. En face OCT and microperimetry may then be beneficial, in addition to conventional FAF which simply shows RPE changes, in identifying the morphological and functional aspects of GA. 61) These findings may be of value when assessing treatments that might prevent or reduce the rate of growth of GA.

An overall and progressive decrease in retinal sensitivity, beyond GA lesions, has been reported. ${ }^{57,60,62}$ Moreover, recent studies, performing a point-to-point correlation of microperimetry and SD-OCT morphology in eyes with GA, precisely correlate single retinal layer defect to retinal sensitivity dysfunction. ${ }^{56,63,64}$ Sayeh et al showed that the largest morphological defect in the area of atrophy graded by SD-OCT was in the photoreceptor layer (external limiting membrane loss and outer plexiform layer thinning and shifting) and not within the RPE layer. Therefore, it may be possible that outer retinal layer loss and disruption precedes the complete loss of the RPE. ${ }^{63}$ Pilotto et al, in a prospective study, detected that microperimetry tested points, showing at baseline the loss of photoreceptor IS/OS junction, had a higher risk of evolving to dense scotoma compared to those with intact photoreceptor IS/OS junction. ${ }^{64}$ Moreover, the same Authors observed that the mean total retinal thickness and the mean outer retinal thickness significantly decreased during follow up, whereas, the mean inner retinal layer thickness (INL) significantly increased. The decrease of the outer retinal layer thickness was inversely correlated to the inner retinal layer thinning (rho $=-0.710$ ). These findings underline that, even if GA is a macular disease that primarily affects the outer retina, the inner retina is also involved. ${ }^{64}$ Retinal remodeling and Müller cells changes and swelling, induced by oxidative stress or preceding degeneration of photoreceptors, have 
been described in an animal model of GA. ${ }^{65-67}$ Müller cell hypertrophy has also been described histologically by Curcio et al. ${ }^{68}$ Because the cell body of Müller cells is located in the inner nuclear layer, Pilotto et al speculated that the inner retinal layer thickening they detected in progressing GA could be caused by Müller cells changes. ${ }^{64}$

With the introduction of multimodal imaging where microperimetry and OCT data are overlapped, it has been possible to hypothesize that different pathophysiologic mechanisms, responsible of GA development and for any progression in AMD, may exist. Recently, it has been observed that patients with bilateral GA and those with GA in one eye and CNV in the fellow eye (unilateral GA) differ for both retinal sensitivity and choroidal thickness changes. ${ }^{69}$ In patients with unilateral GA, choroid was thinner compared to patients with bilateral GA, and became thinner during follow-up. Otherwise, patients with bilateral GA had a significant reduction in retinal sensitivity from baseline, whereas retinal sensitivity did not significantly change in patients with unilateral GA. It may be hypothesized that in patients with GA and CNV in the fellow eye, the development and progression of GA is primarily due to a choroidal disease, and silent CNV cannot be excluded. On the contrary, photoreceptors are primarily involved in patients with bilateral GA, and the progression of the GA probably depends on their dysfunction, with secondary decrease of visual function. ${ }^{69}$

\section{Exudative age-related macular degeneration}

Visual impairment in eyes with choroidal neovascularization (CNV) due to AMD is associated with progressive deterioration not only of visual acuity, but also of retinal fixation and macular sensitivity, because of the development of CNV. This functional deterioration manifests as decreased fixation stability, loss of central fixation, and impaired retinal sensitivity with the development of dense scotomas as detected by microperimetry In eyes with progressive visual function deterioration, the inability to maintain a preferential fixation location within the foveal area progresses until complete absence of foveal (visual) perception is established and the PRL becomes totally eccentric. ${ }^{70-72}$ Eccentric fixation develops early in exudative AMD: a functional marker compared to GA. ${ }^{45,48,72}$

In exudative AMD, fixation patterns deteriorate with increasing duration of symptoms; time is a critical factor in macular diseases from a functional point of view. As CNV develops, patients are initially able to maintain a central and stable fixation. In eyes with subfoveal CNV due to AMD, Fujii et al observed that fixation, assessed by means of SLO microperimetry was central in $75 \%$ of eyes, poorly central in $15 \%$, and eccentric only in $9 \%$ of the cases. ${ }^{70}$ It was stable in $42 \%$, relatively unstable in $39 \%$, and unstable in $18 \%$ of the cases. But, if the duration of symptoms was $<3$ months, fixation pattern was even better; it was central and stable respectively in 89 and in $58 \%$ of the cases. ${ }^{70}$ Using mesopic microperimetry, Midena et al reported a higher rate of eyes with unstable and eccentric fixation in patients affected by AMD with subfoveal CNV with symptoms lasting $>9$ months. A relatively unstable or unstable fixation was present respectively in 35.6 and $38.4 \%$ of the cases. Fixation was poorly central and predominantly eccentric respectively in 15.1 and $63 \%$ of the cases. Fixation stability was also related to best corrected visual acuity. No statistically significant difference in location and stability was observed between classic and occult $\mathrm{CNV} .^{72}$

PRL even if eccentric, may be stable, particularly in patients with longer duration of symptoms. ${ }^{72}$ This means that the visual system needs time to develop an extrafoveal, but stable PRL. As in eyes with advanced GA, (also in) eyes with exudative AMD PRL also tends to be localized in preferred sites of the retina. ${ }^{72-76}$

In patients with exudative AMD the presence of several anatomic abnormalities, including neurosensory retinal detachment, subretinal hemorrhage, RPE hyperplasia, RPE atrophy or detachment and the CNV itself, determines a reduction of retinal sensitivity or a dense scotoma. The size of the absolute scotoma correlates with reading ability and reading speed. ${ }^{76}$ Tezel et al observed that chorioretinal scar, retinal pigment epithelium atrophy, and CNV were the major anatomic lesions correlated with the absolute scotoma at microperimetry. ${ }^{71}$ The presence of an absolute scotoma over CNV well correlates with a previous histopathologic finding, which revealed that $52 \%$ of the photoreceptors over the site of the lesion show extensive degeneration. ${ }^{77}$

The density of the scotoma also correlates with the duration of symptoms. Midena et al detected that a dense scotoma was present in $63.4 \%$ of eyes with exudative AMD, which is lower than that reported by Fujii et al, who detected an absolute scotoma in $28 \% .^{70,72}$ The main difference between the two studies is the duration of symptoms: Midena el al analyzed fixation pattern in AMD patients with symptoms lasting for $>9$ months while on the contrary in the Fujii et al study, 93\% of the patients had symptoms lasting $<3$ months. ${ }^{70,72}$

The density of the scotoma correlates as well with the type of $\mathrm{CNV}$ but only in the early phases of exudative AMD. Classic CNV correlates with a denser scotoma than occult $\mathrm{CNV} .{ }^{78}$ In advanced neovascular AMD there is no statistically significant difference between type of $\mathrm{CNV}$ and presence of dense scotoma. ${ }^{72} \mathrm{~A}$ different pattern of macular functional impairment seems to affect patients with a peculiar neovascular lesion called retinal angiomatous proliferation (or deep retinal vascular 
abnormality or chorioretinal anastomoses). In these lesions, a dense scotoma, migration and instability of fixation all develop in early phases of the disease. The development of a neovascular net in the inner layers of the retina may be the reason for very early impairment of photoreceptors.

The visual system is a plastic neuronal system, which may be reorganized after the deactivation of some of its components. When both eyes are functionally involved by the development of a dense central scotoma, the pattern of fixation and the perception of the scotoma changes. Doris et al, analyzing the relation between macular morphology and visual function in AMD patients with $\mathrm{CNV}$, observed that larger lesion size, greater area of classic $\mathrm{CNV}$, and a greater distance to healthy retina were associated with poorer distance VA and worse contrast sensitivity. ${ }^{79}$ However, when eyes were subdivided into two groups depending on whether the study eye was the better or the worse eye, only the size of the classic component was significantly correlated with near and distance VA. When the study eye was the worse eye different associations were noted. The overall lesion size, closely followed by the distance to healthy retina, showed the strongest correlation with measures of visual function. This means that in the worst eye visual function more closely correlats with the morphologic damage to the macula than it may be expected in the better eye, where the visual function is better than expected for a given macular disease. Therefore, in neovascular AMD, lesions of similar morphology (even at OCT examination), may have a different impact on visual function depending on whether the eye is the better or the worse of the pair. ${ }^{79}$ Similar findings have been described in patients affected by serpiginous choroiditis. ${ }^{47}$ In patients with both eyes affected, a stable and central fixation can be detected in the eye with the better visual acuity, even if the atrophic chorioretinal lesion, due to the progression of the disease, are morphologically similar in both eyes, with an eccentric and unstable fixation in the other eye. It seems that an eye never reaches its full potential unless forced to by loss of the fellow eye. This explains the filling-in phenomenon observed in late AMD. ${ }^{80}$ This phenomenon has been described as a filling-in of the missing information when part of an image falls on a blind area of the visual field. ${ }^{81}$ In AMD patients, the filling-in phenomenon which depends on the presence of a scotoma, it is not seen in eyes whose fellow eye has good vision, and it is only seen in the preferred eye of patients with bilateral macular disease. ${ }^{80}$

\section{Exudative Age-related macular degeneration: treatment}

In the past, detection of retinal fixation by means of SLO microperimetry was proposed before laser photocoagulation of well defined juxtafloveal or extrafoveal CNV secondary to AMD. Manual overlapping of SLO static microperimetry to fluorescein angiography was also performed. After laser treatment most eyes demonstrated foveal fixation unless the fovea was involved by laser scar or its enlargement. ${ }^{82}$ Some studies were also performed in neovascular AMD eyes treated with photodynamic therapy (PDT). A significant benefit of PDT in the preservation of the central visual field was reported using SLO microperimetry. ${ }^{83}$ The final scotoma size was significantly smaller in the PDT group than in the placebo one. In eyes with a foveal PRL at baseline, the threshold of retinal sensitivity at the fovea improved after therapy, resulting in visual acuity improvement. On the contrary, in eyes with a parafoveal or unstable PRL before treatment, a dense scotoma persisted despite complete resolution of exudative manifestations following treatment.

The current approach for the treatment of exudative AMD involves the use of locally administered intravitreal antiangiogenic drugs (bevacizumab, pegaptanib, ranibizumab and aflibercept), whose effects on retinal sensitivity have been more recently investigated. ${ }^{84-86}$

Bolz et al, investigating morphologic and functional effects of the recommended loading regimen with intravitreal ranibizumab, observed significant changes in morphology and function only between baseline and week 1 , with no significant additional morphologic or functional benefit following the second and the third monthly injections. ${ }^{87}$ Longer term functional results were documented by Parravano et al ${ }^{88,89}$ In a retrospective 24-weeks follow-up study, evaluating functional changes after intravitreal ranibizumab (loading phase regimenthree monthly injections-followed by retreatment if signs of activity where still present), these Authors detected that mean retinal sensitivity significantly improved from $3.89 \pm 3.0 \mathrm{~dB}$ at baseline to $6.61 \pm 3.4 \mathrm{~dB}$ even at 24 weeks $(P=0.044)$. Mean visual acuity significantly improved from 48.67 ETDRS letters \pm 8.58 to $60.72 \pm 16.09(P=0.026)$. Improvement in fixation stability from baseline was also observed in $33.3 \%$ of treated eyes. The same Authors confirmed their functional findings after 24 months. ${ }^{88,89}$ Moreover, although visual acuity and retinal thickness changes had their peak 4 weeks after treatment, Parravano et al observed that retinal sensitivity shows more long term progressive improvement. After 24 months of follow-up, intravitreal injections of antiVEGF determined progressive improvement of retinal sensitivity up to the last examination, whereas visual acuity improvement stopped at 6 months, suggesting that microperimetry provide additional information about macular function on a long term basis. ${ }^{88,89}$ The functional discrepancies reported by these two groups may be explained by the different component of the CNV 
included in each study. In Bolz et al series, 31\% of the CNV were predominantly classic, $38 \%$ minimally classic and 31\% occult. Conversely, in Parravano et al series, $22.2 \%$ of CNV were predominantly classic, $16.6 \%$ minimally classic, and $61.1 \%$ occult. The higher rate of predominantly or minimally classic CNV included by Bolz et al may have negatively influenced final functional improvement. ${ }^{87-89}$

With the introduction of spectral domain OCT and the possibility to register the retinal sensitivity at each stimulus location and to correlate it to the corresponding location on SD-OCT morphology, it has been observed that IS/OS junction integrity and external limiting membrane restoration are both strongly positively correlated with the change of retinal sensitivity during treatment with intravitreal drugs. ${ }^{90-92}$

Surgical treatment of neovascular AMD has also been investigated by microperimetry showing poor fixation after submacular surgery. ${ }^{93}$ However, preoperative irreversible damage to the neurosensory retina and intraoperative damage deriving from the separation of $\mathrm{CNV}$ from the neurosensory retina cannot be distinguished, due to the fact that the fovea was relocated over an area surgically devoid of any pigmentation. ${ }^{94}$

\section{Low-vision rehabilitation}

The reduced visual acuity in patients with late stage AMD leads to the loss of reading ability. Therefore, reading ability restoration is one of the main objectives in lowvision rehabilitation (LVR) secondary to advanced AMD. Adaptive strategies to reduce the impact of disabilities due to vision loss develop naturally and quite early. ${ }^{95}$ One such strategy in AMD patients is the attempt to reduce the impact of scotoma on central fields of vision, developing scotoma awareness and displacement abilities. To obtain it, images need to be projected on eccentric PRL, which possess superior visual capabilities and assume the functions of the lost macular area. ${ }^{96}$

One of the basic tenets of modern LVR is the concept of PRL assuming 'macular function' after central vision loss. However, in about 25\% of cases PRL is located onto an 'unfavorable' retinal area and hence it is useless. In addition to traditional low-vision aids and reading rehabilitation training, a new method for PRL rehabilitation is the biofeedback training using the microperimeter. ${ }^{97}$ The biofeedback technique with MP-1 provides an effective method for PRL rehabilitation, by training fixation accuracy and stability, and refixation precision skills. ${ }^{98}$ Microperimetry in fact allows for the investigation of the site and for the characteristics of the PRL. Shima et al observed that in AMD eyes, PRL and highest retinal sensitivity loci, identified by mean of microperimetry, are two separate entities with no identical retinal location. ${ }^{99}$ The aim of LVR interventions should be relocation of PRL to the retinal loci with highest retinal sensitivity, identified by means of microperimetry, because reading speed improves if a new PRL is established in an area that is more favorable for reading. ${ }^{73,99}$

Moreover, microperimetry assesses the site and the characteristic of PRL before and after rehabilitation. Greater fixation stability with better PRL at the end of vision rehabilitation, explains the improvement of visual efficiency.

\section{Conclusion}

Microperimetry provides more information about visual functional changes in patients with AMD than could be obtained by the simple evaluation of visual acuity or other visual function tests.

Visual function deterioration in AMD is characterized by initial progressive deterioration in central retinal sensitivity. Longer duration of the disease is a key factor associated with worse fixation patterns and retinal sensitivity deterioration. A better understanding of the characteristics of visual loss in AMD will aid the clinician in monitoring the natural history of the disease and in quantifying the beneficial or detrimental effects of any therapeutic intervention for AMD.

\section{Conflict of interest}

The authors declare no conflict of interest.

\section{References}

1 Wu Z, Guymer RH, Finger RP. Low luminance deficit and night symptoms in intermediate age-related macular degeneration. Br J Opthalmol 2016; 100: 395-398.

2 Scilley K, Jackson GR, Cideciyan AV, Maguire MG, Jacobson SG, Owsley C. Early age-related maculopathy and self-reported visual difficulty in daily life. Ophthalmology 2002; 109: 1235-1242.

3 Owsley C, McGwin G, Jackson GR, Kallies K, Clark M. Cone- and Rod- mediated dark adaptation impairment in age-related maculopathy. Am J Ophthalmol 2007; 114: 1728-1735.

4 Curcio CA, Owsley C, Jackson GR. Spare the rods, save the cones in aging and age-related maculopathy. Invest Ophthalmol Vis Sci 2000; 41: 2015-2018.

5 Pilotto E, Midena E. Scanning laser microperimetry. In: Midena Eed. Perimetry and the Fundus: An Introduction to Microperimetry. Slack Incorporated: Thorofare, NJ, 2007; 7-12.

6 Midena E, Radin PP, Convento E. Liquid crystal display microperimetryIn:Midena Eeditor. Perimetry and the Fundus: An Introduction to Microperimetry. Slack incorporated: Thorofare, NJ, 2007; 15-25.

7 Weingessel B, Sacu S, Vecsei-Mailovits PV, Weingessel A, Richter-Mueksch S, Schmidt-Erfurth U. Interexaminar and 
intraexaminar reliability of the microperimeter MP-1. Eye 2009; 23: 1052-1058.

8 Rohrschneider K, Springer C, Bultmann S, Volcker HE. Microperimetry-comparison between the micro perimeter 1 and scanning laser ophthalmoscope-fundus perimetry. Am J Ophthalmol 2005; 139: 125-134.

9 Midena E, Vujosevic S, Cavarzeran FMicroperimetry Study Group. Normal values for fundus perimetry with the micrperimeter MP1. Ophthalmology 2010; 117: 1571-1576.

10 Midena E, Convento E, Radin PP, Cavarzeran F. Macular automatic fundus perimetry threshold versus standard perimetry threshold. EJO 2007; 17: 65-68.

11 Vujosevic S, Midena E, Pilotto E, Radin PP, Chiesa L, Cavarzeran F. Diabetic macular edema: correlation between microperimetry and Optical Coherence Tomography findings. Invest Ophthalmol Vis Sci 2006; 47: 3044-3051.

12 Markowitz SN, Reyes SV. Microperimetry and clinical practice: an evidence-based review. Can J Ophthalmol 2013; 48: 350-357.

13 Fujii GY, de Juan E, Sunness JS, Humayum MS, Pieramici DJ, Chang TS. Patient selection for macular translocation surgery using the scanning laser ophthalmoscope. Ophthalmology 2002; 109: 1737-1744.

14 Timberlake GT, Sharma MK, Grose SA, Gobert DV, Gauch JM, Maino JH. Retinal location of the preferred retina locus relative to the fovea in scanning laser ophthalmoscope images. Optom VisSci 2005; 82: 177-185.

15 Longhin E, Convento E, Pilotto E, Bonin G, Vujosevic S, Kotsafti $\mathrm{O}$ et al. Statitc and dynamic retinal fixation stability in microperimetry. Can J Ophthalmol 2013; 48: 375-380.

16 Wu Z, Ayton LN, Guymer RH, Luu CD. Intrasession test-retest variability of microperimetry in age-related macular degeneration. Invest Ophthalmol Vis Sci. 2013; 54: 7378-7385.

17 Vujosevic S, Smolek MK, Lebow KA, Notaroberto N, Pallikaris A, Casciano M. Detection of macular function changes in early (AREDS 2) and intermediate (AREDS 3) age-related macular degeneration. Ophthalmologica 2011; 225 155-160.

18 Weiter J, Delori FC, Wing GL, Fitch KA. Retinal pigment epithelial lipofuscin and melanin and choroidal melanin in human eyes. Invest Ophthalmol Vis Sci. 1986; 27: 145-152.

19 Delori FC, Dorey CK, Staurenghi G, Arend O, Goger DG, Weiter JJ. In vivo fluorescence of the ocular fundus exhibits retinal pigment epithelium lipofuscin characteristics. Invest Ophthalmol Vis Sci 1995; 36: 718-729.

20 Owsley C, Jackson GR, Cideciyan AV, Huang Y, Fine SL, Ho AC et al. Psychophysical evidence for rod vulnerability in age-related macular degeneration. Invest Ophthalmol Vis Sci. 2000; 41: 267-273.

21 Midena E, Segato T, Blarzino MC, Degli Angeli C. Macular drusen and the sensitivity of the central visual field. Doc Ophthalmol 1994; 88: 179-185.

22 Steinmetz RL, Haimovici R, Jubb C, Fitzke FW, Bird AC. Syntomatic abnormalities of dark adaptation in patients with age-related Bruch's membrame change. Br J Ophthalmol 1993; 77: 549-554.

23 Eisner A, Stoumbos VD, Klein ML, Fleming SA. Relations between fundus appearance and function. Eyes whose fellow eye has exudative age-related macular degeneration. Invest Ophthalmol Vis Sci 1991; 32: 8-20.

24 Smith CV, Pokorny J, Diddie KR. Color matching and Stiles-Crawford effect in observers with early age-related macular changes. J Opt Soc Am A 1988; 5: 2113-2121.
25 Johnson PT, Lewis GP, Talaga KC, Brown MN, Kappel PJ, Fisher SK et al. Drusen-associated degeneration in the retina. Invest Ophthalmol Vis Sci 2003; 44: 4481-4488.

26 Johnson PT, Brown MN, Pulliam BC, Anderson DH, Johnson LV. Synaptic pathology, altered gene expression, and degeneration in photoreceptors impacted by drusen. Invest Ophthalmol Vis Sci 2005; 46: 4788-4795.

27 Curcio CA, Medeiros NE, Millican CL. Photoreceptor loss in age-related macular degeneration. Invest Ophthalmol Vis Sci 1996; 37: 1236-1249.

28 Schuman SG, Koreishi AF, Farsiu S, Jung S, Izatt JA, Toth CA. Photoreceptor layer thinning over drusen in eyes with age-related macular degeneration imaged in vivo with Spectral-Domain optical coherence tomography. Ophthalmology 2009; 116: 488-496.

29 Parisi V, Perillo L, Tedeschi M, Scassa C, Gallinaro G, Capaldo $\mathrm{N}$ et al. Macular function in eyes with early age-related macular degeneration with or without controlateral late age-related macular degeneration. Retina 2007; 27: 879-890.

30 Midena E, Vujosevic S, Convento E, Manfrè A, Cavarzeran F, Pilotto E. Microperimetry and fundus autofluorescence in patients with early age-related macular degeneration. $\mathrm{Br} J$ Ophthalmol 2007; 91: 499-503.

31 Hartmann KI, Bartsch D-U, Cheng L, Kim JS, Gomez ML, Klein $\mathrm{H}$ et al. Scanning laser ophthalmoscope imaging stabilized microperimetry in dry age related macular degeneration. Retina 2011; 31: 1323-1331.

32 Wu Z, Ayton LN, Luu CH, Guymer RH. Longitudinal changes in Microperimetry and low luminance visual acuity in age-related macular degeneration. JAMA Ophthalmol 2015; 133: $442-448$.

33 Chandramohan A, Stinnett SS, Petrowski JT, Schuman SG, Toth CA, Cousins SW et al. Visual function measures in early and intermediate age-related macular degeneration. Retina 2016; 36: 1021-1031.

34 Sivaprasad S, Bird A, Nitiahpapand R, Nicholson L, Hykin P, Chatziralli I. Perspectives on reticular pseudodrusen in age-related macular degeneration. Surv Ophthalmol 2016; 61(5): 521-537.

35 Spaide RF. Outer retinal atrophy after regression of subretinal drusenoid deposit as a newly recognized form of late age-related macular degeneration. Retina 2013; 33(9): 1800-1808.

36 Hogg RE, Silva R, Staurenghi G, Murphy G, Santos AR, Rosina $C$ et al. Clinical characteristics of reticular pseudodrusen in the fellow eye of patients with unilateral neovascular age-related macular degeneration. Ophthalmology 2014; 121(9): 1748-1755.

37 Ooto S, Ellabban AA, Ueda-Arakawa N, Oishi A, Tamura H, Yamashiro $\mathrm{K}$ et al. Reduction of retinal sensitivity in eyes with reticular pseudodrusen. Am J Ophthalmol. 2013; 156(6): 1184-1191.

38 Querques G, Massamba N, Srour M, Boulanger E, Georges A, Souied EH. Impact of reticular pseudodrusen on macular function. Retina 2014; 34(2): 321-329.

39 Steinberg JS, Fitzke FW, Fimmers R, Fleckenstein M, Holz FG, Schmitz-Valckenberg S. Scotopic and photopic Microperimetry in patients with reticular drusen and age-related macular degeneration. JAMA Ophthalmol 2015; 133: 690-697.

40 Klein R, Klein BE, Knudtson MD, Meuer SM, Swift M, Gangnon RE. Fifteen-year cumulative incidence of 
age-related macular degeneration: the Beaver Dam Eye Study. Ophtahlmology 2007; 114: 253-262.

41 Sunness JS, Gonzales-Baron J, Applegate CA, Bressler NM, Tian Y, Hawkins B et al. Enlargement of atrophy and visual acuity loss in the geographic atrophy form of age-related macular degeneration. Ophthalmology 1999; 106: 1768-1779.

42 Sunness JS, Margalit E, Srikurnaran D, Applegate CA, Tian Y, Perry D et al. The long-term natural history of geographic atrophy from age-related macular degeneration. Ophthalmology 2007; 114: 271-277.

43 Schatz H, McDonald H. Atrophic macular degeneration: rate of spread of geographic atrophy and visual loss. Ophthalmology 1989; 96: 1541-1551.

44 Holz FG, Bindewald-Wittich A, Fleckenstein M, Dreyhaupt J, Scholl HP, Schmitz-Valckenberg $S$ et al. Progression of geographic atrophy and impact of fundus autofluorescence patterns in age-related macular degeneration. Am J Ophthalmol 2007; 143: 463-472.

45 Sunness JS, Bressler NM, Mahuire MG. Scanning laser ophthalmoscopic analysis of the pattern of visual loss in age-related geographic atrophy of the macula. Am J Ophthalmol 1995; 119: 143-151.

46 Schmitz-Valckenberg S, Fleckenstein M, Scholl HPN, Holz F. Fundus autofluorescence and progression of age-related macular degeneration. Surv Ophthalmol 2009; 54: 96-117.

47 Pilotto E, Vujosevic S, Grgic VA, Sportiello P, Convento E, Secchi AG et al. Retinal function in patients with serpiginous choroiditis: a microperimetry study. Graefes Arch Clin Exp Ophthalmol. 2010; 248: 1331-1337.

48 Sunness JS, Applegate CA, Haselwood D, Rubin GS. Fixation patterns and reading rates in eyes with central scotomas from advanced atrophic age-related macular degeneration and stargardt disease. Ophthalmology 1996; 103: 1458-1466.

49 Sunness JS, Applegate CA. Long-term follow-up of fixations patterns in eyes with central scotoma from geographic atrophy associated with age-related macular degeneration. Am J Ophthalmol 2005; 140: 1085-1093.

50 Holz FG, Bellmann C, Margaritidis M, Otto TP, Völcker HE. Patterns of increased in vivo fundus autofluorescence in the junctional zone of geographic atrophy of the retinal pigment epithelium associated with age-related macular degeneration. Graefes Arch Clin Exp Ophthalmol 1999; 237: 145-152.

51 von Ruckmann A, Fitzke FW, Bird AC. Distribution of fundus autofluorescence with a scanning laser Ophthalmoscope. Br J Ophthalmol 1995; 79: 407-412.

52 Keilhauer CN, Delori FC. Near-infrared autofluorescence imaging of the fundus: visualization of ocular melanin. Invest Ophthalmol Vis Sci 2006; 47: 3556-3564.

53 Pilotto E, Vujosevic S, Melis R, Convento E, Sportiello P, Alemany-Rubio $\mathrm{E}$ et al. Short wavelength fundus autofluorescence versus near-infrared fundus autofluorescence, with microperimetric correspondence, in patients with geographic atrophy due to age-related macular degeneration. Br J Ophthalmol 2011; 95: 1140-1144.

54 Sholl HP, Bellman CDS, Bird AC, Fitzke FW. Photopic and scotopic fine matrix mapping of retinal areas of increased fundus autofluorescence in patients with age-related macular degeneration. Invest Ophthalmol Vis Sci 2004; 45: 574-583.

55 Schmitz-Valckenberg S, Bültmann S, Dreyhaupt J, Bindewald A, Holz FG, Rohrschneider K. Fundus autofluorescence and fundus perimetry in the junctional zone of geographic atrophy in patients with age-related macular degeneration. Invest Ophthalmol Vis Sci 2004; 45: $4470-4476$

56 Querques L, Querques G, Forte R, Souied EH. Microperimetric correlations of autofluorescence and optical coherence tomography imaging in dry age-related macular degeneration. Am J Opthalmol 2012; 153: 1110-1115.

57 Pilotto E, Guidolin F, Convento E, Spedicato L, Vujosevic S, Cavarzeran $\mathrm{F}$ et al. Fundus autofluorescence and microperimetry in progressing geographic atrophy secondary to age-related macular degeneration. $\mathrm{Br} \mathrm{J}$ Ophthalmol. 2013; 97: 622-626.

58 Pilotto E, Guidolin F, Convento E, Antonini R, Stefanon FG, Parrozzani R et al. En face Optical Coherence Tomography to detect and measure geographic atrophy. Invest Ophthalmol Vis Sci 2015; 56: 8120-8124.

59 Portella Nunes R, Gregori G, Yehoshua Z, Stetson PF, Feuer W, Moshfeghi AA et al. Predicting the Progression of Geographic Atrophy in Age-Related Macular Degeneration With SD-OCT En Face Imaging of the Outer Retina. Ophthalmic Surg Lasers Imaging Retina 2013; 44: 344-359.

60 Hariri AH, Tepelus TC, Akil H, Nittale MG, Sadda SR. Retinal sensitivity at the junctional zone of eyes with geographic atrophy due to age-related macular degeneration. Am J Ophthalmol 2016; 168: 122-128.

61 Pilotto E, Convento E, Guidolin F, Abalsamo CK, Longhin E, Parrozzani $\mathrm{R}$ et al. Microperimetry features of geographic atrophy identified with en face optical cohenrence tomography. JAMA Ophthalmol 134(8): 873-879.

62 Meleth AD, Mettu P, Agrón E, Chew EY, Sadda SR, Ferris FL et al. Changes in Retinal Sensitivity in Geographic Atrophy Progression as Measured by Microperimetry. Invest Ophthalmol Vis Sci 2011; 52: 1119-1126.

63 Sayegh RG, Kiss CG, Simader C, Kroisamer J, Montuoro A, Mittermuller TJ et al. A systematic correlation of morphology and function using spectral domain optical coherence tomography and microperimetry in patients with geographic atrophy. Br J Ophtalmol 2014; 98: 1050-1055.

64 Pilotto E, Benetti E, Convento E, Guidolin F, Longhin E, Parrozzani R et al. Microperimetry, fundus autofluorescence, and retinal layer changes in progressing geographic atrophy. Can J Ophthalmol. 2013; 48: 386-393.

65 Hariri S, Moayed AA, Choh V, Bizheva K. In vivo assessment of thickness and reflectivity in a rat outer retinal degeneration model with ultrahigh resolution optical coherence tomography. Invest Ophthalmol Vis Sci. 2012; 53: 1982-1989.

66 Marc RE, Jones BW, Watt CB, Vazquez-Chona F, Vaughan DK, Organisciak DT. Extreme retinal remodeling triggered by light damage: implications for age related macular degeneration. Mol Vis. 2008; 14: 782-806.

67 DiLoreto Jr DA, Martzen MR, del Cerro C, Coleman PD, del Cerro M. Müller cell changes precede photoreceptor cell degeneration in the age-related retinal degeneration of the Fischer 344 rat. Brain Res. 1995; 698: 1-14.

68 Curcio CA, Messinger JD, Sloan KR, Mitra A, McGwin G, Spaide RF. Human chorioretinal layer thicknesses measured in macula-wide, high-resolution histologic sections. Invest Ophthalmol Vis Sci. 2011; 52: 3943-3954.

69 Pilotto E, Guidolin F, Convento E, Stefanon FG, Parrozzani R, Midena E. Progressing geographic atrophy: choroidal thickness and retinal sensitivity identify two clinical phenotypes. Br J Ophthalmol 2015; 99: 1082-1086. 
70 Fujii Gy, De Juan Jr E, Humayun MS, Sunness JS, Chang TS, Rossi JV et al. Characteristics of visual loss by scanning laser opthalmoscope microperimetry in eyes with subfoveal choroidal neovascularization secondary to age-related macular degeneration. Am J Ophthalmol 2003; 136: 1067-1078.

71 Tezel TH, Del Priore L, Flowers BE, Grosof DH, Benenson IL, Zamora RL et al. Correlation between scanning laser ophthalmoscope microperimetry and anatomic abnormalities in patients with subfoveal neovascularization. Ophthalmology 1996; 103: 1829-1836.

72 Midena E, Radin PP, Pilotto E, Ghirlando A, Convento E, Varano $\mathrm{M}$ et al. Fixation pattern and macular sensitivity in eyes with subfoveal choroidal neovascularization secondary to age-related macular degeneration. A microperimetry study. Sem Ophthalmol 2004; 19: 55-61.

73 Mori F, IshiKo S, Kitaya N, Takamiya A, Sato E, Hikichi T et al. Scotoma and fixation patterns using scanning laser ophthalmoscope microperimetry in patients with macular dystrophy. Am J Ophthalmol 2001; 132: 897-902.

74 Acosta F, Lashkari K, Reynaud X, Jalkh AE, Van de Velde F, Chedid N. Characterization of function changes in macular holes and cysts. Ophthalmology 1991; 98: 1820-1823.

75 Guez Je, Le Gargasson JF, Rigaudiere F, O'Regan JK. Is there a systematic location for the pseudofovea in patients with central scotoma? Vision Res 1993; 33: 1271-1279.

76 Fletcher DC, Schuchard RA. Preferred retinal loci relationship to macular scotomas in a low vision population. Ophthalmology 1997; 104: 632-638.

77 Green WR, Enger C. Age-related macular degeneration histopathologic studies: the 1992 Lorenz E. Zimmerman Lecture. Ophthalmology 1993; 100: 1519-1535.

78 Schneider U, Inhoffen W, Gelisken F, Kreissig I. Assessment of visual function in choroidal neovascularization with scanning laser microperimetry and simultaneous indocyanine green angiography. Graefe's Arch Clin Exp Ophthalmol 1996; 234: 612-617.

79 Doris N, Hart PM, Chakravarthy U, McCleland J, Stevenson M. Relation between macular morphology and visual function in patients with choroidal neovascularization of age related macular degeneration. Br J Ophthalmol 2001; 85: 184-188.

80 Cohen Sy, Lamarque F, Saucet JC, Provent P, Langram C, LeGargasson JF. Filling-in phenomenon in patients with agerelated macular degeneration: differences regarding uni-or bilaterality of central scotoma. Graefes Arch Clin Exp Ophthalmol 2003; 241: 785-791.

81 Ramachandram VS, Gregory RL. Perceptual filling in of artificially induced scotomas in human vision. Nature 1991; 350: 699-702.

82 Oshima Y, Harino S, Tano Y. Scanning laser ophthalmoscope microperimetric assessment in patients with successful laser treatment for juxtafoveal choroidal neovascularization. Retina 1998; 18: 109-117.

83 Schmidt-Erfurth UM, Michels SM. Changes in confocal ICG angiography through 2 years after photodynamic therapy with verteporfin. Ophthalmology 2003; 110: 1306-1314.

84 Squirrell DM, Mawer NP, Mody CH, Brand CS. Visual outcome after intravitreal ranibizumab for wet age-related macular degeneration: a comparison between best-corrected visual acuity and microperimetry. Retina 2010; 30: 436-442.
85 Ozdemir H, Karacorlu M, Senturk F, Karacorlu SA, Uysal O. Microperimetric changes after intravitreal bevacizumab injection for exudative age-related macular degeneration. Acta Ophthalmol. 2012; 90: 71-75.

86 Prager F, Michels S, Simader C, Geitzenauer W, Schmidt-Erfurth UM, Simader C. Changes in retinal sensitivity in patients with neovascular age-related macular degeneration after systemic bevacizumab (Avastin) therapy. Retina 2008; 28: 682-688.

87 Bolz M, Simader C, Ritter M, Ahlers C, Benesch T, Prünte C et al. Morphological and functional analysis of the loading regimen with intravitreal ranibizumab in neovascular age-related macular degeneration. Br J Ophthalmol. 2010; 94: 185-189.

88 Parravano MC, Oddone F, Tedeschi M, Chiaravallotti A, Perillo L, Boccassini B et al. Retinal functional changes measured by microperimetry in neovascular age-related macular degeneration patients treated with ranibizumab. Retina 2010; 30: 1017-1024.

89 Parravano M, Oddone F, Tedeschi M, Schiano Lomoriello D, Chiaravalloti A, Ripandelli G et al. Retinal functional changes measured by microperimetry in neovascular age-related macular degeneration patients treated with ranibizumab. Retina 2009; 29: 329-334.

90 Sulzbacher F, Kiss C, Kaider A, Roberts P, Munk M, Kroh ME et al. Correlation of SD-OCT features and retinal sensitivity in neovascular age-related macular degeneration. Invest Ophthalmol Vis Sci. 2012; 53: 6448-6455.

91 Sulzbacher F, Roberts P, Munk MR, Kaider A, Kroh ME, Sacu $S$ et al. Relationship of retinal morphology and retinal sensitivity in the treatment of neovascular age-related macular degeneration using aflibercept. Invest Ophthalmol Vis Sci 2014; 56: 1158-1167.

92 Hartmann KI, Oster SF, Amini P, Bartsch DU, Cheng L, Freeman WR. SLO-Microperimetry in Wet Age-Related Macular Degeneration During Anti-VEGF Therapy. Ophthalmic Surg Lasers Imaging Retina 2015; 46: 824-830.

93 Loewenstein A, Sunness SS, Bressler NM, Maarsh MJ, de Juan E Jr. Scanning laser ophthalmoscope fundus perimetry after surgery for choroidal neovascularization. Am J Ophthalmol 1998; 125: 657-665.

94 Chieh JJ, Stinnett SS, Toth CA. Central and pericentral retinal sensitivity after macular translocation surgery. Retina 2008; 28: 1522-1529.

95 Cheung SH, Legge GE. Functional and cortical adaptations to central vision loss. Vis Neurosci 2005; 22: 187-201.

96 White JM, Bedell HE. The oculomotor reference in humas with bilateral macular disease. Invest Ophthalmol Vis Sci 1990; 31: 1149-1161.

97 Giorgi D, Contestabile MT, Pacella E, Gabrieli CB. An instrument for biofeedback applied to vision. Appl Psychophysiol Biofeedback 2005; 30: 389-395.

98 Tarita-Nistor L, Gonzalez E, Markowitz SN, Steinbach M. Plasticity of fixation in patients with central vision loss. Vis Neurosci 2009; 26: 487-494.

99 Shima N, Markowitz SN, Reyes Sv. Concept of a functional retinal loss in age-related macular degeneration. Can J Ophthalmol 2010; 45: 62-66. 